\title{
Dexamethasone Acetate
}

National Cancer Institute

\section{Source}

National Cancer Institute. Dexamethasone Acetate. NCI Thesaurus. Code C1067.

The acetate salt form of Dexamethasone, a synthetic adrenal corticosteroid with potent anti-inflammatory properties. In addition to binding to specific nuclear steroid receptors, dexamethasone also interferes with NF-kB activation and apoptotic pathways. This agent lacks the salt-retaining properties of other related adrenal hormones. ( $\mathrm{NCl04)}$ 